\title{
nab-Paclitaxel in Combination with Carboplatin for a Previously Treated Thymic Carcinoma
}

\author{
Go Makimoto Keiichi Fujiwara Hiromi Watanabe \\ Nobuhisa Kameyama Mizuho Matsushita Kammei Rai Ken Sato \\ Toshiro Yonei Toshio Sato Takuo Shibayama \\ Department of Respiratory Medicine, National Hospital Organization Okayama Medical \\ Center, Okayama, Japan
}

\section{Key Words}

Thymic carcinoma $\cdot$ nab-Paclitaxel $\cdot$ Carboplatin $\cdot$ Chemotherapy

\begin{abstract}
We present the case of a 40-year-old man with previously treated thymic carcinoma, complaining of gradually worsening back pain. Computed tomography scans of the chest showed multiple pleural disseminated nodules with a pleural effusion in the right thorax. The patient was treated with carboplatin on day 1 plus nab-paclitaxel on day 1 and 8 in cycles repeated every 4 weeks. Objective tumor shrinkage was observed after 4 cycles of this regimen. In addition, the elevated serum cytokeratin 19 fragment level decreased, and the patient's back pain was relieved without any analgesics. Although he experienced grade 4 neutropenia and granulocyte colony-stimulating factor (G-CSF) injection, the severity of thrombocytopenia and nonhematological toxicities such as reversible neuropathy did not exceed grade 1 during the treatment. To our knowledge, this is the first report to demonstrate the efficacy of combination chemotherapy consisting of carboplatin and nab-paclitaxel against thymic carcinoma. This case report suggests that nab-paclitaxel in combination with carboplatin can be a favorable chemotherapy regimen for advanced thymic carcinoma.
\end{abstract}


Makimoto et al:: nab-Paclitaxel in Combination with Carboplatin for a Previously

\section{Introduction}

Thymic epithelial neoplasm is a relatively rare malignant disease arising from the thymic epithelium and comprises thymoma, thymic carcinoma, and thymic neuroendocrine carcinoma. The incidence of thymic carcinoma is much lower than that of thymoma, accounting for $1-4 \%$ of anterior mediastinal tumors, and has a high risk of invasion and metastasis [1]. The standard regimen of chemotherapy for thymic carcinoma has not yet been determined because of its rarity, although a combination chemotherapy that comprised (solvent-based) paclitaxel and carboplatin was recently reported to have favorable antitumor activity for advanced thymic carcinoma [2,3]. We present the case of a previously treated thymic carcinoma where favorable antitumor effects were achieved by nabpaclitaxel in combination with carboplatin.

\section{Case Presentation}

A 40-year-old man was admitted to our hospital in May 2013 because of progressive right-sided back pain. The patient had been surgically diagnosed with squamous cell carcinoma of the thymus in July 2009 (designated as stage II according to the Masaoka classification [4]) and had received postoperative thoracic irradiation. However, computed tomography (CT) scans of the chest in May 2010 showed pleural dissemination in the right thorax. The patient worked as an engineer and requested to avoid paclitaxel-induced neuropathy at that time. Therefore, he received combined chemotherapy consisting of cisplatin and docetaxel, and achieved a minor response. After the patient became refractory to this regimen, various antitumor agents, including gemcitabine, $S-1$, and amrubicin, were administered. However, pleural dissemination recurred and the patient's back pain gradually worsened, even while using opioids.

Upon admission to our hospital, CT scans of the chest showed multiple pleural disseminated nodules with a pleural effusion in the right thorax (fig. 1a). The serum cytokeratin 19 fragment (CYFRA) level was increased $(13.9 \mathrm{ng} / \mathrm{ml}$; normal level $<3.5 \mathrm{ng} / \mathrm{ml})$. The patient received $n a b$-paclitaxel in combination with carboplatin as described in a previous phase III trial [5]. He experienced grade 4 neutropenia, according to the NCI-CTCAE (National Cancer Institute - Common Terminology Criteria for Adverse Events) version 4.0, 2 weeks after beginning chemotherapy. Therefore, the nab-paclitaxel administration was omitted on day 15 , and the patient received granulocyte colony-stimulating factor (G-CSF) for several days. The severity of thrombocytopenia and nonhematological toxicities such as reversible neuropathy did not exceed grade 1 during the treatment. Objective tumor shrinkage was obtained after 4 cycles of chemotherapy, and the pleural effusion disappeared (fig. 1b). The serum CYFRA level decreased to $1.7 \mathrm{ng} / \mathrm{ml}$, and the patient's back pain was relieved without any opioids.

\section{Discussion}

nab-Paclitaxel, the 130-nm albumin-bound paclitaxel formulation, is a promising new agent for non-small cell lung cancer, breast cancer, and gastric cancer. This case report suggests that $n a b$-paclitaxel, when combined with carboplatin, is also a promising therapy for thymic carcinoma. To our knowledge, this is the first report to demonstrate the efficacy of combination chemotherapy consisting of carboplatin and nab-paclitaxel against thymic 
Makimoto et al.: nab-Paclitaxel in Combination with Carboplatin for a Previously Treated Thymic Carcinoma

carcinoma. Additionally, this regimen seems to be active against previously treated or refractory thymic carcinoma. In a published series, a combination chemotherapy that comprised (solvent-based) paclitaxel and carboplatin was reported to have favorable antitumor activity and a response rate in approximately one-third of patients with thymic carcinoma [2]. Considering its efficacy against thymic carcinoma, nab-paclitaxel in combination with carboplatin could possibly be expected to achieve tumor regression in this case.

In a preclinical study, intratumoral accumulation of nab-paclitaxel was reported to be $33 \%$ higher than that of solvent-based paclitaxel, indicating that $n a b$-paclitaxel accumulated more effectively within tumors [6]. Recently, weekly nab-paclitaxel plus carboplatin resulted in a significantly improved overall response rate, compared with that of solvent-based paclitaxel plus carboplatin, in non-small cell lung cancer treatment [5]. In particular, patients with squamous cell histology responded remarkably well to treatment with $n a b$-paclitaxel, with a $68 \%$ improvement rate over that in the solvent-based paclitaxel arm, suggesting that $n a b$-paclitaxel is a favorable agent for a squamous subset. The most common histologic type of thymic carcinoma in Japan is squamous cell carcinoma [7]. Therefore, $n a b$-paclitaxel in combination with carboplatin is considered an optional regimen for thymic carcinoma.

\section{Conclusion}

According to our experience, a case of advanced thymic carcinoma responded to treatment with $n a b$-paclitaxel and carboplatin, which can be a useful chemotherapy regimen for thymic carcinoma.

\section{Disclosure Statement}

The authors have no conflicts of interest.

\section{References}

1 Weiss GJ: Thymic carcinoma: current and future therapeutic interventions. Expert Opin Investig Drugs 2010;19:1007-1016.

2 Furugen M, Sekine I, Tsuta K, et al: Combination chemotherapy with carboplatin and paclitaxel for advanced thymic cancer. Jpn J Clin Oncol 2011;41:1013-1016.

-3 Igawa S, Murakami H, Takahashi T, et al: Efficacy of chemotherapy with carboplatin and paclitaxel for unresectable thymic carcinoma. Lung Cancer 2010;67:194-197.

4 Masaoka A, Monden Y, Nakahara K, et al: Follow-up study of thymomas with special reference to their clinical stages. Cancer 1981;48:2485-2492.

-5 Socinski MA, Bondarenko I, Karaseva NA, et al: Weekly nab-paclitaxel in combination with carboplatin versus solvent-based paclitaxel plus carboplatin as first-line therapy in patients with advanced non-smallcell lung cancer: final results of a phase III trial. J Clin Oncol 2012;30:2055-2062.

6 Desai N, Trieu V, Yao Z, et al: Increased antitumor activity, intratumor paclitaxel concentrations, and endothelial cell transport of cremophor-free, albumin-bound paclitaxel, ABI-007, compared with cremophor-based paclitaxel. Clin Cancer Res 2006;12:1317-1324.

7 Kondo K, Monden Y: Therapy for thymic epithelial tumors: a clinical study of 1,320 patients from Japan. Ann Thorac Surg 2003;76:878-884. 
Makimoto et al.: nab-Paclitaxel in Combination with Carboplatin for a Previously Treated Thymic Carcinoma
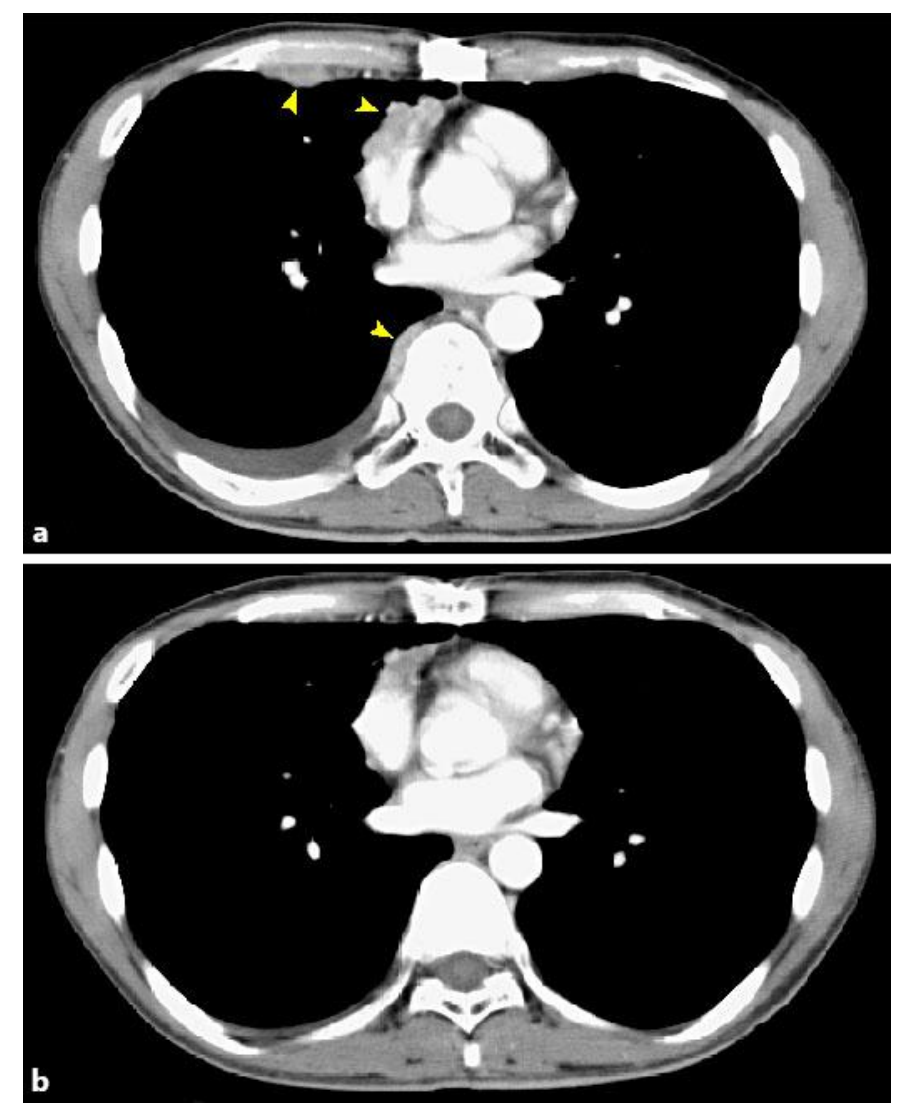

Fig. 1. CT scans of the chest upon admission showed multiple disseminated nodules (indicated by arrowheads) with a pleural effusion in the right thorax (a). Tumor shrinkage was achieved and the pleural effusion disappeared after 4 cycles of $n a b$-paclitaxel plus carboplatin (b). 